\title{
Protein Purification and Western Blot Detection from Single Zebrafish Embryo
}

\author{
Denhi Schnabel, Jorge Castillo-Robles, and Hilda Lomeli
}

\begin{abstract}
Characterization of a protein of interest during development is essential for functional studies. A general strategy for understanding the function of a particular protein involves the generation of null mutations, or treatment with drugs, that interfere with its activity. To demonstrate that the synthesis, stability, or activity of a protein has been affected, accurate and efficient detection of low amounts of protein is essential. This can be achieved by immunohistochemistry or by western blot. Here we describe a method for the detection of proteins from single de-yolked zebrafish embryos. This procedure includes a fixation step and the concomitant elimination of lipids from the yolk cell. We show that this approach allows the rapid analysis of proteins in embryos without having to manually remove the yolk. This method provides a convenient alternative for genotyping of mutant embryos as early as the 128 cell stage. In addition, in drug- or morpholino-treated embryos, the correlation between the penetrance of a phenotype and the concentration of a protein present may be established.
\end{abstract}

Keywords: protein purification, western blot, zebrafish embryo

\section{Introduction}

$\mathbf{Z}$ EBRAFISH HAS BEEN used for decades as an excellent model organism. Its use has increased exponentially with the characterization of novel genes involved in vertebrate development and disease. Every functional study eventually involves the demonstration of differential expression or activity of a protein of interest. The presence or absence of the protein can be determined either by immunohistochemistry or gel electrophoresis followed by a western blot. Successful detection of a target protein depends on the affinity and specificity of the corresponding antibody and on the abundance of the protein of interest. Standard western blots in zebrafish are achieved by pooling tens to thousands of embryos. ${ }^{1}$ Owing to natural variability between individuals with a mutation or after treatment with an inhibitor, a gradient of phenotypes can be observed; when embryos with variable phenotypes are pooled and further analyzed by western blot, the specific amount of a protein in an embryo cannot be correlated with the particularities of its phenotype. Another common problem inherent to zebrafish embryos is the abundance of yolk proteins that interfere with the detection of the protein of interest; when the yolk is not completely removed the sensitivity for detecting other cellular proteins is limited. ${ }^{2}$ Although other methods of protein extraction of single embryos or larvae have been described, they either manually remove the yolk, ${ }^{3}$ which is time consuming, or the yolk is not removed. ${ }^{4,5}$ We have adapted a method whereby the whole protein extracts can be obtained from a single embryo with the concomitant easy removal of yolk. This procedure eliminates the lipids from the sample by using a mix of heptane and methanol, which forms a double layer where the lipids rest, whereas the yolk-free embryos remain at the bottom from where an efficient extraction of proteins is achieved. By using this procedure, we obtained protein from single embryos at different embryonic stages from 128-cells to 5 days postfertilization (dpf) being able to identify proteins present in the nuclei, cytoplasm, and membrane. Moreover, we showed that this protocol is reproducible and it is useful for associating a distinctive phenotype to the absence of protein produced in embryos in CRISPR-Cas9 mutants

\section{Materials and Methods}

\section{Fish maintenance and strains}

AB wild-type zebrafish (Danio rerio) embryos and wildtype embryos from an undefined strain sourced from a local pet store and inbred for several generations were obtained from natural crosses and raised at $28^{\circ} \mathrm{C}$. smarcel mutant crosses were obtained as described previously. ${ }^{6}$ 
Zebrafish embryos were incubated at $28^{\circ} \mathrm{C}$ in embryo water until reaching the desired developmental stage. Embryo stages were determined by morphological criteria according to Kimmel et al. ${ }^{7}$ Zebrafish were handled in compliance with local animal welfare regulations and approved by the Institute's Ethics Committee (Instituto de Biotecnología, UNAM).

\section{smarce1 mutants and genotyping}

smarce 1 mutants were previously generated and described in Castillo-Robles et al. ${ }^{6}$ Embryos were obtained by crossing smarce ${ }^{+/-}$adults; embryos were incubated at $28^{\circ} \mathrm{C}$ in embryo water until $5 \mathrm{dpf}$. Larvae were anesthetized with tricaine, immobilized with methylcellulose on agar plates and visualized with a stereomicroscope (Leica MZ 12.5), and photographed using a CCD camera (AxioCam MRc 5; Zeiss) and the AxioVision Rel. 48 software.

The posterior part of the larvae was used for genotyping; a sharp cut with an insulin needle was performed at the posterior end of the yolk extension, whereas the anterior part was used for protein purification. The primers $5^{\prime}$-TCTGGCCTACAA CAACTACA-3' and 5'-TCCTCTCGGCTGGGCTTTTA- $3^{\prime}$ were used to amplify a $121 \mathrm{bp}$ fragment, and polymerase chain reaction products were separated on $14 \%$ polyacrylamide gels where an 8-bp deletion can be distinguished. ${ }^{8}$

\section{Total protein preparation and western blotting}

Total protein was obtained from single zebrafish embryos with the protocol modified form Sue. ${ }^{9}$ In brief, individual embryos or larvae are gently pipetted in a $1.5 \mathrm{~mL}$ eppendorf tube. The embryo water is discarded completely and $500 \mu \mathrm{L}$ of heptane is added, immediately after $500 \mu \mathrm{L}$ of cold methanol is added, and samples are fixed for $5 \mathrm{~min}$ with gentle shaking and rinsed twice with $500 \mu \mathrm{L}$ of cold methanol. Embryos or larvae were washed twice with $100 \mu \mathrm{L}$ of cold embryo buffer (Tris $10 \mathrm{mM} \mathrm{pH} \mathrm{7.5,} \mathrm{sodium} \beta$-glycerophosphate $80 \mathrm{mM}$ pH7.5, ethylene glycol-bis(2-aminoethylether)$\mathrm{N}, \mathrm{N}, \mathrm{N}^{\prime}, \mathrm{N}^{\prime}$-tetraacetic acid (EGTA) $20 \mathrm{mM}, \mathrm{MgCl}_{2} 15 \mathrm{mM}$, $\mathrm{Na}_{3} \mathrm{VO}_{4} 2 \mathrm{mM}$, benzamidine $1 \mathrm{mM}$, sodium metabisulfite $1 \mathrm{mM}$, phenylmethylsulfonyl fluoride (PMSF) $2 \mathrm{mM}$, and Tween-20 $0.05 \%$ ), each sample was suspended in $20 \mu \mathrm{L}$ of

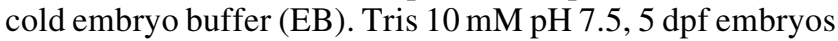
were homogenized with a small plastic pestle in $30 \mu \mathrm{L}$ of cold EB. An aliquot was obtained to quantify total protein with the Bradford assay. $5 \times$ sodium dodecyl sulfate (SDS) sample buffer was added and boiled for $5 \mathrm{~min}$. The western blotting was performed as previously described ${ }^{10}$ with some modifications. The antibodies used were anti-E-cadherin (610182, AB_397581; BD Biosciences), anti-Erk-1 (sc153; Santa Cruz Biotechnology), anti-SMARCE1 antibody (Abcam ab131328), and the secondary antibody used was anti-rabbit IgG-HRP (sc2004; Santa Cruz Biotechnology).

\section{Results}

Total proteins extracts were obtained at several distinctive embryonic stages, from 128-cell stage to $5 \mathrm{dpf}$ (Fig. 1A). To demonstrate that this method is suitable to detect proteins with different cellular localization from single embryos, we used antibodies for the identification of E-cadherin, a cell adhesion protein located in the membrane; Erk1 a serine/ threonine kinase present in the cytoplasm and Smarce1, a
A

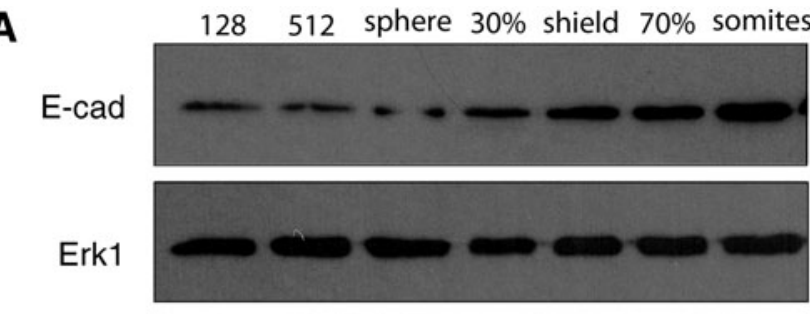

Smarce 1

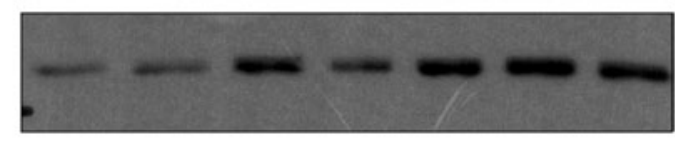

B
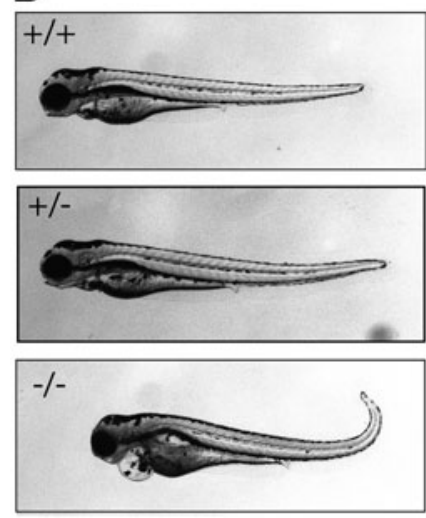

C

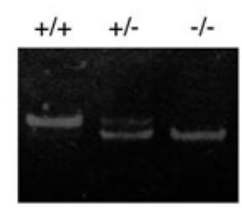

D

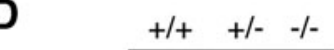

Smarce1

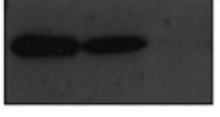

Erk1

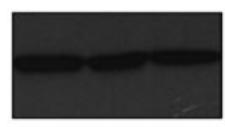

FIG. 1. Single embryo protein purification. (A) Western blot of single proteins purified from embryos at different stages 128,512 , sphere, $30 \%$ epiboly, shield, $70 \%$ epiboly, and 18 somites. The use of antibodies for E-cadherin (E-cad), Erk1, and Smarce1 proteins is indicated (representative blots are depicted here). (B) Wild-type $(+/+)$, heterozygous $(+/-)$, and homozygous embryos. The characteristic phenotype of the smarce 1 mutants is observed with a cardiac edema, and the body curvature. (C) Genotypes of polymerase chain reaction smarcel embryos. A $121 \mathrm{bp}$ amplicon, including the mutated region, is obtained from the wild-type embryos while a 113 fragment is amplified from the mutated allele. (D) Western blot with the Smarce1 antibody from $4 \mathrm{dpf}$ wildtype single embryos (+/+) and normal looking heterozygous embryos (+/-) derived from smarcel (+/-) in crosses and their edematous curved siblings (-/-). Erk1 protein was used as a control to confirm that the same amount of protein was loaded in each well (representative blots are depicted here).

subunit of the BAF chromatin remodeling complex, that acts in the nuclei. These three proteins were successfully detected in all the embryonic stages tested (Fig. 1A).

Next, we evaluated this technique for the characterization of the phenotypes produced in embryos with mutations. For this purpose, we isolated protein from single embryos of smarce 1 mutants previously described. ${ }^{6}$ smarcel mutants exhibit very distinctive defects at 72 hours postfertilization, including smaller eyes, abnormal body curvature, and heart abnormalities. We previously showed that mutant embryos have heart defects, including dysmorphic chambers, absence of looping, slower beating, and absence of circulation; these defects were accompanied with an abnormal morphology of the endocardium. ${ }^{6}$ Since smarce 1 mutants are easily distinguished by their phenotype, larvae can be separated into groups, defective and normal phenotype (Fig. 1B). After 
taking photographs of each larva, the posterior part of the larva was used to genotype and larvae were classified as wildtype, heterozygous, and homozygous larvae (Fig. 1C). The anterior part of each larva was used to obtain total protein. The cell lysates were loaded in an SDS-polyacrylamide gel electrophoresis (SDS-PAGE) gel and blotted onto a nitrocellulose membrane to be analyzed by western blot against Smarce1 and as a loading control we included Erk1 (Fig. 1D). We were able to demonstrate that larvae with a distinctive phenotype were those in which Smarce1 could not be detected, whereas wild type and heterozygotes presented the expected levels of Smarce1 protein (Fig. 1D), with heterozygous embryos showing a lower concentration of protein compared with the homozygous wild-type embryo.

In conclusion, we have adapted a protocol to identify proteins from yolk-free single embryos at different stages of development, which is less time consuming, reproducible, and permits many biological and experimental replicates to be performed.

\section{Acknowledgments}

We thank Dulce I. Pacheco-Benítez for technical support with the animal care. We thank Dr. Christopher Wood for the careful reading of the article.

\section{Disclosure Statement}

No competing financial interests exist.

\section{Funding Information}

This study was supported by grants given to D.S. (IN200618).

\section{References}

1. Westerfield M. The Zebrafish Book. A Guide for the Laboratory Use of Zebrafish (Danio rerio), 4th ed., Univ. of Oregon Press, Eugene, 2000.

2. Link V, Shevchenko A, Heisenberg CP. Proteomics of early zebrafish embryos. BMC Dev Biol 2006;6:1.
3. Campbell WA, Yang H, Zetterberg H, Baulac S, Sears JA, Liu T, et al. Zebrafish lacking Alzheimer presenilin enhancer 2 (Pen-2) demonstrate excessive p53-dependent apoptosis and neuronal loss. J Neurochem 2006;96:1423-1440.

4. Choorapoikayil S, Weijts B, Kers R, de Bruin A, den Hertog J. Loss of Pten promotes angiogenesis and enhanced vegfaa expression in zebrafish. Dis Model Mech 2013;6: 1159-1166.

5. Dupret B, Völkel P, Follet P, Le Bourhis X, Angrand PO. Combining genotypic and phenotypic analyses on single mutant zebrafish larvae. Methods X 2018;5:244-256.

6. Castillo-Robles J, Ramírez L, Spaink HP, Lomelí H. smarcel mutants have a defective endocardium and an increased expression of cardiac transcription factors in zebrafish. Sci Rep 2018;8:15369.

7. Kimmel CB, Ballard WW, Kimmel SR, Ullmann B, Schilling TF. Stages of embryonic development of the zebrafish. Dev Dyn 1995;203:253-310.

8. Chen J, Zhang X, Wang T, Li Z, Guan, G, Hong Y. Efficient detection, quantification and enrichment of subtle allelic alterations. DNA Res 2012;19:423-433.

9. Su TT. Immunoblotting of proteins from single Drosophila embryos, chapter 34. In: Drosophila Protocols Drosophila Melanogaster. Sullivan W, Ashburner M, and Hawley RS (eds), pp. 577-583, CSHL Press, New York, 2000.

10. Miranda-Rodriguez JR, Salas-Vidal E, Lomeli H, Zurita M, Schnabel D. RhoA/ROCK pathway activity is essential for the correct localization of the germ plasm mRNAs in zebrafish embryos. Dev Biol 2017;421:27-42.

Address correspondence to: Denhi Schnabel, PhD

Instituto de Biotecnología

UNAM

Avenida Universidad \#2001, Colonia Chamilpa, Apdo.

Postal 510-3

Cuernavaca C.P. 62250

Morelos

Mexico

E-mail: denhi@ibt.unam.mx 